
.

ISSN: 2578-0379

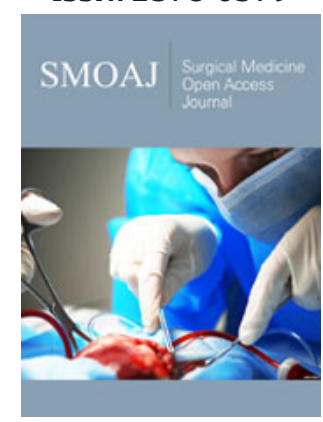

*Corresponding author: Lobanov IE, Doctor of Technical Sciences, Moscow

Submission: :-lin November 20, 2020

Published: 毕December 09, 2020

Volume 4 - Issue 1

How to cite this article: Lobanov IE. Low-Reynolds Mathematical Modeling of the Structure of Vortex Zones Between Periodic Surface-Located Flow Turbulators of Semicircular Cross-Section in Pipes. Surg Med Open Acc J. 4(1). SMOAJ.000579. 2020. DOI: 10.31031/SMOAJ.2020.04.000579

Copyright@ Lobanov IE, This article is distributed under the terms of the Creative Commons Attribution 4.0 International License, which permits unrestricted use and redistribution provided that the original author and source are credited.

\section{Low-Reynolds Mathematical Modeling of the Structure of Vortex Zones Between Periodic Surface-Located Flow Turbulators of Semicircular Cross-Section in Pipes}

\author{
Lobanov IE* \\ Doctor of Technical Sciences, Moscow
}

\section{Annotation}

Mathematical modeling of the structure of vortex zones between periodic surface-located flow turbulators of a semicircular and square cross-section is carried out on the basis of multi-block computational technologies based on the solution of the factorized finite-volume method of the Reynolds equations (closed with the help of the Menter shear stress transfer mode) and the energy equation (on different-scale intersecting structured grids). This method was previously successfully applied and verified by experiment. An exhaustive analysis of the corresponding streamlines is presented, proving the advantage of abruded turbulators.

A well-known and very well-tested in practice method of vortex intensification of heat transfer is the application of periodic protrusions on the walls of the washed surfaces [1]. The study of the structure of the intensified flow is mainly carried out by experimental methods [1], while modern computational works on this topic are relatively few [2] and are only partially devoted directly to the structure of the intensified flow; some of the methods (for example, a certain part of works [2]) use only integral approaches to this problem. This work is directly devoted to the study of the flow structure in the pipe, For pipes with turbulators of a semicircular cross-section, the nature of the relationship between heat transfer and hydraulic resistance remains similar to the above-considered nature of the relationship for square flow turbulators, but the values $(\mathrm{Nu} / \mathrm{Nusm}) /(\xi / \xi \mathrm{sm})$ for the former, as a rule, is definitely higher due to the much smaller influence of the systems of secondary and corner vortices, which occurs due to deformation and greater elongation of the main vortex, which is confirmed by the corresponding values for pipes with turbulators of a semicircular cross-section $(\mathrm{Nu} /$ Nusm) $/(\xi / \xi \mathrm{sm})=0.89$ for $\mathrm{t} / \mathrm{D}=1.00, \mathrm{~d} / \mathrm{D}=0.94, \mathrm{Re}=104 ;(\mathrm{Nu} / \mathrm{Nusm}) /(\xi / \xi \mathrm{sm})=0.68$ for $\mathrm{d} / \mathrm{D}=0.94, \mathrm{t} / \mathrm{D}=0.50, \mathrm{Re}=104 ;(\mathrm{Nu} / \mathrm{Nusm}) /(\xi / \xi \mathrm{sm})=0.80$ for d/D=0.94, t/D=0.25, Re=104. Similar conclusions will take place at higher relative heights of turbulators (at $d / D=0.90$ ), but at lower values of the complex $(\mathrm{Nu} / \mathrm{Nusm}) /(\xi / \xi \mathrm{sm})$. For example, for turbulators with a semicircular cross-section, this complex in the considered range of determining parameters $(d / D=0.90 ; t / D=0.25 \div 1.00 ; R e==104 \div 105)$ is of the order of $(\mathrm{Nu} / \mathrm{Nusm}) /$ $(\xi / \xi \mathrm{sm}=0.31 \div 0.50$.

For square turbulators, the above complex is even smaller. The structure of the vortex zones before and after the rib largely depends on its geometry and the coolant flow regime. The prevalence of the generation of turbulence over dissipation occurs mainly at the boundary of the vortex zone remote from the wall, where the velocity gradient, as well as turbulent stresses, are maximum. When intensifying heat transfer by turbulators of square 
cross section, i.e. rather sharp outlines, the energy dissipation that occurs in powerful vortices before and behind them can be comparable to the development of turbulence in them (except for the upper edge of the turbulator), which causes increased hydraulic losses. For pipes with turbulators with a semicircular cross-section, i.e. rather smooth outlines, the above effect is largely neutralized. Consequently, it is more optimal to switch to abruted turbulators (the maximum generation of turbulence in them is located approximately at the level of the upper boundary of the protrusion), where the hydraulic losses are much less, which is confirmed by experimental data.

\section{References}

1. Kalinin EK, Dreitser GA, Kopp IZ (1998) Effective surfaces of heat exchange. Energoatomizdat, p.408.

2. Lobanov IE, NV Paramonov (2011) Mathematical modeling of intensified heat transfer during flow in channels on the basis of complex models of a turbulent boundary layer. Publishing house MAI, p.160.

For possible submissions Click below: 